\title{
Modelagem Matemática do Tempo de Vida de Baterias de Lítio Íon Polímero a partir do Modelo Elétrico para Predizer Runtime e Características V-I
}

\author{
Suelen Cibeli Wottrich ${ }^{1}$ \\ Marcia de Fatima Brondani ${ }^{2}$ \\ Airam Teresa Zago Romcy Sausen ${ }^{3}$ \\ Paulo Sérgio Sausen ${ }^{4}$ \\ Departamento de Ciências Exatas e Engenharias, Programa de Pós-Graduação Stricto Sensu em \\ Modelagem Matemática, Unijuí, Ijuí-RS
}

Resumo. Neste trabalho é realizada a modelagem matemática do tempo de vida de baterias do tipo Lítio Íon Polímero (LiPo), utilizando o modelo elétrico para Predizer Runtime e Características V-I (i.e., de tensão e corrente). A estimação dos parâmetros deste modelo é feita a partir de uma metodologia baseada em ajuste de curvas. As simulações computacionais são realizadas com o auxílio do software Matlab, e os dados experimentais utilizados na parametrização e na validação do modelo são coletados de uma plataforma de testes. O resultados obtidos pelo modelo são satisfatórios, apresentando erros inferiores ao limite considerado aceitável para a classe de modelos elétricos.

Palavras-chave. Modelagem Matemática, Modelo Elétrico para Predizer Runtime e Características V-I, Tempo de Vida de Baterias

\section{Introdução}

Os dispositivos móveis, devido à sua ampla mobilidade e facilidade de uso, tiveram nos últimos anos um crescente avanço em sua comercialização. Associado a este crescimento, modificaram-se algumas de suas funcionalidades, provocando aumento do consumo de energia, bem como, da expectativa do tempo de vida da bateria que abastece o seu sistema operacional [1]. A necessidade de promover o desenvolvimento de baterias mais eficientes, seguras e com maior durabilidade, se torna evidente diante deste contexto. Assim, a realização de pesquisas, que visam o estudo a respeito do comportamento dinâmico das baterias, são de fundamental importância para o desenvolvimento de métodos capazes de prever o seu tempo de vida. Este, por sua vez é definido como o intervalo de tempo transcorrido, durante o processo de descarga, até que seja alcançado um determinado nível inferior de carga (i.e., nível de Cutoff) [10].

\footnotetext{
${ }^{1}$ suwottrich@hotmail.com

${ }^{2}$ brondani.marcia@gmail.com

3 airam@unijui.edu.br

${ }^{4}$ sausen@unijui.edu.br
} 
Uma maneira de predizer o tempo de vida de baterias é através da aplicação de modelos matemáticos que simulam o processo de descarga de energia nas baterias $[1-6,8,9]$. Neste contexto, o Grupo de Automação Industrial e Controle - GAIC, da Universidade Regional do Noroeste do Estado do Rio Grande do Sul - UNIJUÍ, tem realizado diversas pesquisas e aplicações de modelos matemáticos capazes de predizer o tempo de vida de baterias de dispositivos móveis. Nas pesquisas realizadas pelo GAIC, pretende-se verificar qual modelo matemático é o melhor para descrever o comportamento dinâmico do processo de descarga de uma bateria [5].

O modelo elétrico para Predizer Runtime e Características V-I se destaca por possuir alta acurácia na predição do tempo de vida de baterias, conseguindo capturar as características elétricas e dinâmicas, tais como, a tensão em circuito aberto, a capacidade utilizável e a resposta transiente [2]. Algumas técnicas para a estimação dos parâmetros deste modelo são encontradas na literatura, dentre elas destaca-se a técnica de estimação de parâmetros por meio de ajustes de curvas, utilizada por Chen e Rincón-Mora [2].

O presente trabalho propõe a realização da modelagem matemática do tempo de vida de baterias de LiPo, modelo PL383562-2C, a partir do modelo elétrico para Predizer Runtime e Características V-I, sendo aplicada uma técnica baseada em ajuste de curvas para a estimação de seus parâmetros. As simulações computacionais são realizadas com o auxílio do software Matlab, e a validação do modelo é realizada a partir da comparação entre os resultados simulados e os dados experimentais, obtidos de uma plataforma de testes.

O restante deste artigo está estruturado da seguinte forma. Na Seção 2 é apresentado o modelo para Predizer Runtime e Características V-I. Na Seção 3 é apresentada a estimação de parâmetros realizada a partir de uma metodologia baseada em ajustes de curvas. Na Seção 4 são apresentados os resultados das simulações e análise. E, na Seção 5 é apresentada a conclusão.

\section{Modelo Elétrico para Predizer Runtime e Características V-I}

O modelo elétrico para Predizer Runtime e Características V-I prevê simultaneamente o tempo de vida da bateria, a resposta transiente e o estado estacionário de maneira acurada, intuitiva e abrangente, capturando as características elétricas e dinâmicas das baterias, tais como, a capacidade utilizável, a tensão em circuito aberto e a resposta transiente $[2,9]$. Os circuitos $R C$ que compõem este modelo estão dispostos na Figura 1.

$\mathrm{O}$ circuito $R C$ do lado esquerdo é responsável por modelar a capacidade, o $S O C$ e o tempo de vida da bateria [2]. Desta forma, a resistência de autodescarga $R_{\text {self-discharge }}$ é utilizada para caracterizar a perda de energia da célula e a tensão $V_{S O C}$ representa o SOC da bateria variando de $0 V(S O C$ de $0 \%)$ a $1 V(S O C$ de $100 \%)$. Por sua vez, o circuito $R C$ do lado direito simula a resposta transiente e as características de tensão e corrente (V-I) da bateria, em que a tensão da fonte controlada é utilizada para relacionar o SOC com a tensão em circuito aberto $V_{O C}[7]$.

A carga total armazenada na bateria é representada pelo capacitor carregado $C_{\text {capacity }}$, 

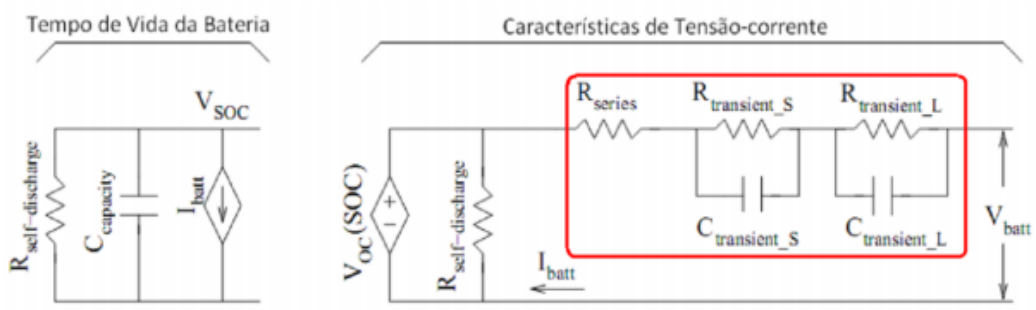

Figura 1: Modelo elétrico para Predizer Runtime e Características V-I [9].

cujo valor é dado a partir da conversão da capacidade nominal da bateria de $A h$ para Columb, conforme a equação (1),

$$
C_{\text {capacity }}=3600 \text { Capacity } f_{1}(\text { ciclo }) f_{2}(\text { temp }),
$$

onde: Capacity é a capacidade nominal em Ah, e f1(ciclo) e f2(temp) são fatores de correção que dependem do número de ciclos e da temperatura $[2,9]$.

O $S O C$ é obtido pela equação,

$$
S O C(t)=S O C_{\text {inicial }}-\frac{\int I_{\text {batt }}(t)}{C_{\text {capacity }}}
$$

onde: $S O C_{\text {inicial }}$ representa o estado de carga incial da bateria e $I_{\text {batt }}$ é a corrente de descarga aplicada a ela.

O tempo de vida da bateria é obtido quando a tensão da bateria atinge a tensão final de descarga. Assim, a tensão do modelo pode ser representada por

$$
V_{\text {batt }}(t)=V_{O C}(S O C)-I_{\text {batt }} R_{\text {series }}-V_{\text {transient }}(t),
$$

onde: $V_{\text {batt }}$ representa a tensão final da bateria, $V_{O C}(S O C)$ é a tensão em circuito aberto, $R_{\text {series }}$ é a resistência em série e $V_{\text {transient }}$ é a tensão transiente [4]. Os elementos $V_{O C}(S O C)$ e $R_{\text {series }}$ são funções de $S O C$, determinadas pelas equações

$$
\begin{gathered}
V_{O C}(S O C)=a_{0} e^{-a_{1} S O C}+a_{2}+a_{3} S O C-a_{4} S O C^{2}+a_{5} S O C^{3}, \\
R_{\text {series }}(S O C)=b_{0} e^{-b_{1} S O C}+b_{2},
\end{gathered}
$$

A tensão transiente $V_{\text {transient }}$ é dada por

$$
V_{\text {transient }}(t)=V_{\text {transient_S }}(t)+V_{\text {transient_L }}(t),
$$

onde: $V_{\text {transient_S }}(t)$ é a tensão transiente de curta duração descrita pela equação (7) e $V_{\text {transient_L }}(t)$ é a tensão de longa duração descrita pela equação (8) [4].

$$
V_{\text {transient_S }}(t)=\left\{\begin{array}{cc}
R_{\text {transient_S }} I_{\text {batt }}\left[1-e^{-\frac{\left(t-t_{0}\right)}{\tau S}}\right], & t_{0}<t<t_{d} \\
V_{\text {transient_S }} \cdot e^{-\frac{\left(t-t_{d}\right)}{\tau S}}, & t_{d}<t<t_{r}
\end{array}\right.
$$


onde: $R_{\text {transient_S }}$ é a resistência transiente de curta duração, $V_{\text {trasient_S }}$ é a tensão transiente de curta duração no tempo final de descarga, $\tau S=R_{\text {transient_S }} C_{\text {transient_S }}$ e $C_{\text {transient_S }}$ é a capacitância transiente de curta duração [4].

$$
V_{\text {transient_L }}(t)=\left\{\begin{array}{cc}
R_{\text {transient_L }} I_{\text {batt }}\left[1-e^{-\frac{\left(t-t_{0}\right)}{\tau L}}\right], & t_{0}<t<t_{d} \\
V_{\text {transient_L }} \cdot e^{-\frac{\left(t-t_{d}\right)}{\tau L}}, & t_{d}<t<t_{r}
\end{array}\right.
$$

onde: $R_{\text {transient_L }}$ é a resistência transiente de longa duração, $V_{\text {transient_L }}$ é a tensão transiente de longa duração no tempo final de descarga, $\tau L=R_{\text {transient }_{L} L} C_{\text {transient_L }}$ e $C_{\text {transient_L } L}$ é a capacitância transient de longa duração [4].

Os elementos que modelam a tensão transiente também são funções de $S O C$, descritas por

$$
\left\{\begin{array}{l}
R_{\text {transient_S }}(S O C)=c_{0} e^{-c_{1} S O C}+c_{2}, \\
C_{\text {transient_S }}(S O C)=d_{0} e^{-d_{1} S O C}+d_{2}, \\
R_{\text {transient_L }}(S O C)=e_{0} e^{-e_{1} S O C}+e_{2}, \\
C_{\text {transient_L }}(S O C)=f_{0} e^{-f_{1} S O C}+f_{2} .
\end{array}\right.
$$

Destaca-se que os 21 parâmetros das equações (4), (5) e (9) precisam ser estimados e variam de acordo com o tipo de bateria utilizado, considerando as suas propriedades e características. A seguir é apresentada a metodologia adotada para a estimação destes parâmetros.

\section{Estimação dos Parâmetros do Modelo}

Os parâmetros do modelo elétrico para Predizer Runtime e Características V-I são estimados a partir da metodologia de ajuste de curvas, utilizada por Chen e RincónMora [2]. Para a realização desta estimação são utilizados quatro perfis de correntes pulsadas de $80 m A, 160 m A, 320 m A$ e $640 m A$, apresentados na Figura 2. Estes perfis são provenientes de dados coletados em uma plataforma de testes, desenvolvida pelo GAIC e localizada no Laboratório de Sensores Inteligentes da UNIJUÍ.

A metodologia baseada em ajuste de curvas evidenciada no trabalho desenvolvido por [2] consiste em determinar os parâmetros das funções apresentadas nas equações (4), (5), (9). Assim, para cada função é necessário determinar um conjunto de pontos considerando os quatro perfis de descargas pulsadas. Neste contexto, o ajuste de curvas consiste em determinar a curva que passa por estes conjuntos de dados. O método utilizado para o ajuste das curvas é o de Mínimos Quadrados Não Lineares, implementado no software Matlab, obtendo deste modo a equação que representa cada função do modelo com os respectivos parâmetros, os quais estão organizados na Tabela 1.

\section{Resultados das Simulações e Análise}

Nesta seção são apresentados resumidamente os resultados simulados pelo modelo elétrico para Predizer Runtime e Características V-I parametrizado por meio do ajuste de curvas. Os resultados são analisados considerando a diferença entre os tempos de vida experimentais, obtidos na plataforma de testes e, o tempos de vida simulados pelo modelo. 

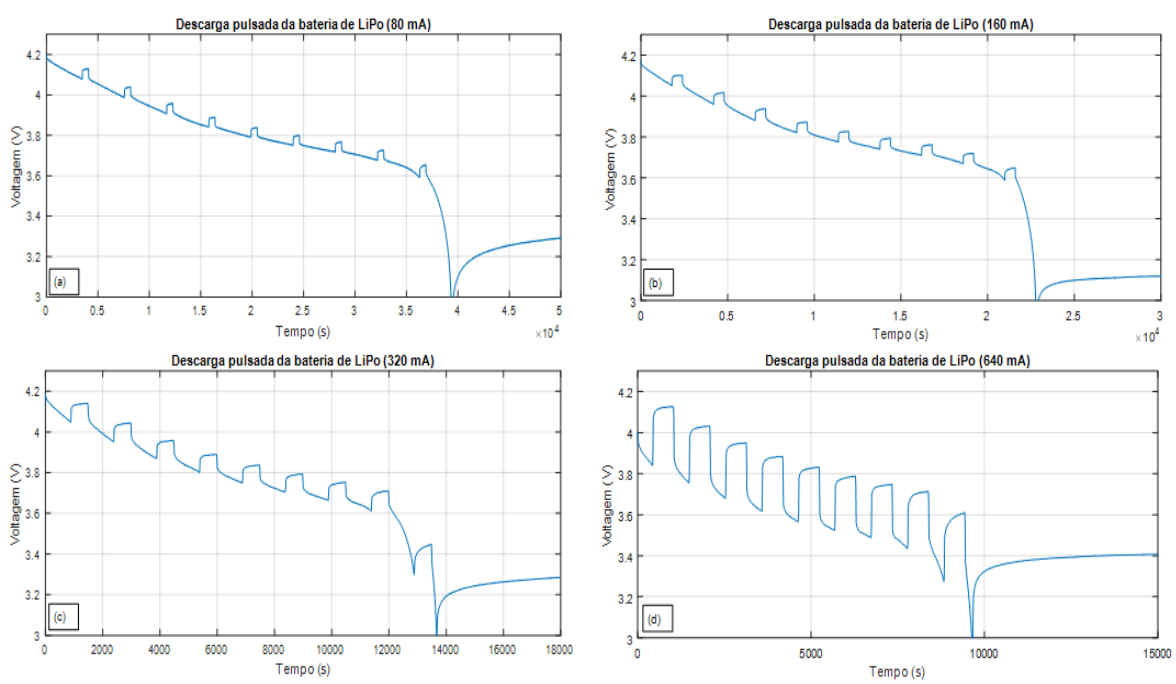

Figura 2: Perfis de descargas pulsadas utilizados para estimação dos parâmetros do modelo.

Tabela 1: Parâmetros do modelo para Predizer Runtime e Características V-I estimados a partir do ajuste de curvas.

\begin{tabular}{|c|c|c|c|c|c|}
\hline a0 & $-1,0931$ & b1 & 41,0174 & d2 & 548,3854 \\
\hline a1 & 23,8482 & b2 & 0,3169 & e0 & 0,3325 \\
\hline a2 & 3,7037 & c0 & 0,5927 & e1 & 4,2892 \\
\hline a3 & 0,0808 & c1 & 18,7453 & e2 & 0,0146 \\
\hline a4 & 0,2980 & c2 & 0,0721 & f0 & $-2342,0565$ \\
\hline a5 & 0,1428 & d0 & $-455,1850$ & f1 & 6,3571 \\
\hline b0 & 1,0632 & d1 & 9,4617 & f2 & 3036,0800 \\
\hline
\end{tabular}

Na Tabela 2 é apresentada a validação da estimação dos parâmetros, que contém os quatro perfis de descargas pulsadas utilizados neste processo com os correspondentes tempos de vida simulados pelo modelo $T V_{\text {sim }}$, tempos de vida experimentais $T V_{\text {exp }}$ e os erros. Também, é exposto o erro médio do modelo para estas simulações, que é de 1,33\%, sendo considerado relativamente baixo.

Tabela 2: Validação da estimação dos parâmetros do modelo.

\begin{tabular}{cccl}
\hline Perfil de Descarga Pulsada $(\boldsymbol{m A})$ & $T V_{\text {sim }}(\boldsymbol{s})$ & $T V_{\text {exp }}(\boldsymbol{s})$ & Erro* $\left.^{*} \boldsymbol{\%}\right)$ \\
\hline 80 & 40390 & 39330 & 2,69 \\
160 & 22790 & 22770 & 0,09 \\
320 & 13990 & 13670 & 2,34 \\
640 & 9610 & 9630 & 0,21 \\
\hline Erro médio & $\mathbf{1 , 3 3}$ \\
\hline & &
\end{tabular}


A validação do modelo é realizada com base em 31 perfis de descargas constantes, com valores entre $50 \mathrm{~mA}$ e $800 \mathrm{~mA}$, com intervalos de $25 \mathrm{~mA}$. Para cada um destes perfis são feitos 8 ensaios na plataforma de testes, e obtido o tempo de vida experimental médio do perfil, utilizado como referência para o cálculo do erro do modelo. Considerando estas simulações, o modelo apresenta o erro médio de $2,22 \%$, sendo considerado um resultado satisfatório, pois está dentro da faixa dos $5 \%$ considerada como adequada para validar os modelos elétricos.

\section{Conclusões}

Neste trabalho, o modelo elétrico para Predizer Runtime e Características V-I foi utilizado para a predição do tempo de vida de baterias de LiPo, modelo PL383562-2C. Para a modelagem matemática foram utilizados dados experimentais do processo real de descarga das baterias, adquiridos em uma plataforma de testes, desenvolvida pelo GAIC. As simulações computacionais foram realizadas no software Matlab e os resultados simulados foram comparados com os resultados experimentais. Os resultados obtidos, tanto para a estimação dos parâmetros quanto para a validação do modelo, foram satisfatórios. Os erros médios obtidos pelo modelo foram válidos para ambos os casos, pois não ultrapassaram o limite de $5 \%$ aceitável para a validação de modelos elétricos.

\section{Referências}

[1] M. Brondani, "Modelagem matemática do tempo de vida de baterias de lítio íon polímero utilizando algoritmos genéticos," Mestrado, Universidade Regional do Noroeste do Estado do Rio Grande do Sul, Ijuí-RS, Fevereiro de 2015.

[2] M. Chen and G. Rincón-Mora, "Accurate electrical battery model capable of predicting runtime and i-v performance,"IEEE Transactions on Energy Conversion, vol. 21, no. 2, pp. 504-511, Junho 2006.

[3] M. Doyle, T. F. Fuller, and J. S. Newman, "Modeling of galvanostatic charge and discharge of the lithium, polymer, insertion cell," Journal of The Electrochemical Society, vol. 140, pp. 1526-1533, 1993.

[4] L. Fransozi, "Modelagem matemática do tempo de vida de baterias de lítio Íon polímero através de modelos híbridos," Mestrado, Universidade Regional do Noroeste do Estado do Rio Grande do Sul, Ijuí-RS, Fevereiro 2015.

[5] D. Freitas, "Estudo e aplicação de modelos analíticos na predição do tempo de vida de baterias utilizadas em dispositivos móveis: Proposição de extensões aos modelos tradicionais," Mestrado, Universidade Regional do Noroeste do Estado do Rio Grande do Sul, Ijuí-RS, Setembro 2015.

[6] M. R. Jongerden and B. Haverkort, "Battery modeling," Thecnical Report in Faculty Electrical Engineering, Janeiro 2008. 
[7] T. KIM, "A hybrid battery model capable of capturing dynamic circuit characteristics and nonlinear capacity effects," Dissertation (Master of Science), University of Nebraska, Lincoln - Nebraska, July 2012.

[8] M. Machado, "Modelagem matemática do tempo de vida de baterias utilizando modelos autorregressivos," Mestrado, Universidade Regional do Noroeste do Estado do Rio Grande do Sul, Ijuí-RS, Abril 2014.

[9] C. Porciuncula, "Aplicação de modelos elétricos de bateria na predição do tempo de vida de dispositivos móveis," Mestrado, Universidade Regional do Noroeste do Estado do Rio Grande do Sul, Ijuí-RS, Abril 2012.

[10] D. Rakmatov, S. Vrudhula, and D. Wallach, "Battery lifetime prediction for energyaware computing,"in Low Power Electronics and Design, 2002. ISLPED'02. Proceedings of the 2002 International Symposium on, 2002, pp. 154-159. 